Available online on 15.09.2017 at http://iddtonline.info
Journal of Drug Delivery and Therapeutics
O 2011-17, publisher and licensee JDDT, This is an Open Access article which permits unrestricted
noncommercial use, provided the original work is properly cited

Open $\odot$ Access

Research Article

\title{
FORMULATION AND EVALUATION OF IN SITU OCULAR GEL OF LEVOFLOXACIN
}

\author{
Prasanth VV ${ }^{1}$, Della Grace Thomas Parambi ${ }^{2}$, Shashi Ranjan ${ }^{3}$ \\ ${ }^{1}$ Professor/Principal, Mount Zion College of Pharmaceutical Sciences and Research, Chayalode, Adoor, Pathanamthitta, Kerala, India, \\ 691556. \\ ${ }^{2}$ Assistant Professor, Department of Pharmaceutical Chemistry, Al Jouf University, P.O. Box 2014, Al Jouf, Sakaka, Saudi Arabia. \\ ${ }^{3}$ Lecturer, Department of Pharmaceutics, St. John's Pharmacy College, No. 6, $9^{\text {th }}$ Cross, $2^{\text {nd }}$ Main, Vijayanagar II Stage, RPC Layout, \\ Bangalore, India,560 040 .
}

\section{ABSTRACT}

Total of 8 formulations of in situ gels of Levofloxacin hydrochloride were prepared by $\mathrm{pH}$ triggered in situ gelling system using different polymers like sodium alginate as gelling agent, Noveon AA-1 polycarbophil and HPMC E50LV as viscosity enhancing agent and benzalkonium chloride as preservative. All the prepared formulations were clear and the visual appearance was found to be transparent. The $\mathrm{pH}$ of the prepared formulations was ranged between 6.50and 7.00. The drug content varied between $96.84 \pm$ 0.396 and $99.65 \pm 0.489 \%$ which indicated that the uniform distribution of drug was found in all the prepared formulations. Among all the formulations, the formulations A4 and A8 showed better gelling capacity. The shear rate on the preparation was large during the blinking stage. From the in vitro drug release profile the formulations A4 and A8 was selected as the best formulations and these formulations were used for further studies such as mechanism of drug release, sterility, antimicrobial efficacy, ocular irritation and accelerated stability. Both formulations provided good fit to the Higuchi model. According to this model, the drug release from these gels may be controlled by diffusion through the micro-pores. The selected formulations showed good anti-microbial action against the organisms and ocular irritation studies revealed that the selected formulations were good with non-irritation and there were no ocular damage or abnormal clinical signs. During and at the end of the accelerated stability study, the selected formulations did not undergo any chemical changes/interaction and remained stable during the study period and showed almost similar physical stability and drug content.

Key Words: Levofloxacin hydrochloride, in situ gels, in vitro drug release, Higuchi model, accelerated stability study.

Article Info: Received 10 July, 2017; Review Completed 30 Aug, 2017; Accepted 31 Aug, 2017; Available online 15 Sep, 2017

\section{Cite this article as:}

Prasanth VV, Parambi DGT, Ranjan S, Formulation and evaluation of in situ ocular gel of levofloxacin, Journal of Drug Delivery and Therapeutics. 2017; 7(5):68-73

DOI: http://dx.doi.org/10.22270/jddt.v7i5.1489

*Address for Correspondence

Shashi Ranjan, Lecturer, Department of Pharmaceutics, St. John's Pharmacy College, No. 6, 9th Cross, 2nd Main, Vijayanagar II Stage, RPC Layout, Bangalore-560 040, E-mail: ranjanshashi20@gmail.com

\section{INTRODUCTION}

Conventional liquid ophthalmic preparations reveal low bioavailability because of a steady lacrimal drainage in the eye. The average drainage of an instilled drug quantity commences instantly upon instillation and is basically accomplished within $5 \mathrm{~min}$. Typical ophthalmic bioavailability $(1-10 \%)$ are achieved owing to the short pre-corneal residence time of ophthalmic solutions. As a result there is a need for recurrent instillation of concentrated solutions to attain the preferred curative effect ${ }^{1}$.

Ocular drug delivery is one of the major challenging and motivating activities which the pharmaceutical scientists are facing now a day, the main difficulty encountered to 
the pharmaceutical scientists is fast pre-corneal removal of the drug, ensuing in deprived bioavailability and healing response because of high lacrimal fluid yield. In situ formed gels are liquid which upon insertion, experience phase conversion in the cul de sac of eye to form a visco-elastic gel and these formed gels tender a reaction to environmental changes. In the last few years, a notable number of $\mathrm{pH}$, novel temperature, and ion induced in situ gel forming systems have been reported for constant ocular delivery of drugs $^{2}$. The reduced bioavailability and curative response showed by conventional ocular solutions due to quick pre-corneal drug elimination may be sweeped over by the use of a gel system which are instilled into the cul de sac as a drop and which undergoes a sol-gel conversion in eye ${ }^{3}$.

LEV, Biopharmaceutical Classification System I, is a broad spectrum anti-infective agent, under the third generation fluoroquinolone derivative mainly used in the infection of the eye such as acute conjunctivitis. The recommended dosage of LEV for the treatment of bacterial conjunctivitis is 1 or 2 drops of $0.5 \%$ solution in the affected eyes for every 2 hours upto 8 times for 2 days, then 1 or 2 drops every 4 hours up to 4 times for next 5 days ${ }^{4}$. LEV is quickly and fully absorbed subsequent to oral administration. Peak plasma concentrations are typically attained one to two hours subsequent to oral dose. The normal terminative plasma elimination half-life of levofloxacin is ranging from around 6 to 8 hours consequent to single or multiple doses of levofloxacin administered either intravenously or orally ${ }^{5}$.

In the present study an endeavor was prepared to develop an in situ ocular gel of LEV to increase ocular contact time, enhance the corneal permeability and site specificity for the better treatment of conjunctivitis and corneal ulceration with reduced adverse effects and better patient compliance.

\section{MATERIALS AND METHODS}

\section{Materials}

Levofloxacin (LEV) was obtained as a gift sample from Caplin Point Laboratories Ltd, Chennai, India. Hydroxypropyl methylcellulose (HPMC E50LV), Noveon AA-1polycarbophil, Sodium alginate, Benzalkonium chloride, Sodium chloride and Sodium hydroxide were purchased from SD Fine Chemicals, Bangalore, India. All the chemicals and reagents used were of analytical grade.

\section{Methods}

\section{Formulation of in situ gels of LEV}

The composition of different formulations of LEV in situ ocular gels is shown in Table 1. The sodium chloride $(0.9 \% \mathrm{w} / \mathrm{v})$ was dissolved in $50 \mathrm{~mL}$ of distilled water and viscosity enhancifier was added to the above solution and stirred slowly with a magnetic stirrer. Care was taken that no lumps were formed during stirring. The polymers were sprinkled over this solution and allowed to hydrate overnight and stirred using a magnetic stirrer. LEV was dissolved in phosphate buffer ( $\mathrm{pH}$ 7.4) and benzalkonium chloride was added and the solution was filtered through $0.2 \mu \mathrm{m}$ cellulose acetate membrane filter. The drug solution was added to the polymeric solution under constant stirring until a uniform solution was obtained. The $\mathrm{pH}$ of the formulation was then adjusted to 6.50 using $0.1 \mathrm{~N}$ $\mathrm{NaOH}$. The developed formulations were filled in glass vials, closed with gray butyl rubber closures and sealed with aluminium caps. The formulations in their final pack were subjected to terminal sterilization by autoclaving at $121^{\circ} \mathrm{C}$ at 15 psi for $20 \mathrm{~min}^{6}$.

Table 1: Formulation of in situ ocular gel of LEV

\begin{tabular}{|l|c|c|c|c|c|c|c|c|}
\hline \multirow{2}{*}{ Compositions } & \multicolumn{9}{|c|}{ Concentration (\% w/v) } \\
\cline { 2 - 10 } & $\mathbf{A 1}$ & $\mathbf{A 2}$ & $\mathbf{A 3}$ & $\mathbf{A 4}$ & $\mathbf{A 5}$ & $\mathbf{A 6}$ & $\mathbf{A 7}$ & $\mathbf{A 8}$ \\
\hline Levofloxacin & 0.5 & 0.5 & 0.5 & 0.5 & 0.5 & 0.5 & 0.5 & 0.5 \\
\hline HPMC E50LV & 0.2 & 0.3 & 0.4 & 0.5 & 0.2 & 0.3 & 0.4 & 0.5 \\
\hline Noveon AA-1 polycarbophil & 0.5 & 0.5 & 0.5 & 0.5 & - & - & - & - \\
\hline Sodium alginate & - & - & - & - & 0.5 & 0.5 & 0.5 & 0.5 \\
\hline Benzalkonium chloride & 0.002 & 0.002 & 0.002 & 0.002 & 0.002 & 0.002 & 0.002 & 0.002 \\
\hline Sodium chloride & 0.9 & 0.9 & 0.9 & 0.9 & 0.9 & 0.9 & 0.9 & 0.9 \\
\hline Sodium hydroxide & q.s. & q.s. & q.s. & q.s. & q.s. & q.s. & q.s. & q.s. \\
\hline Phosphate buffer pH 7.4 (mL) & 100 & 100 & 100 & 100 & 100 & 100 & 100 & 100 \\
\hline
\end{tabular}

\section{Evaluations}

\section{Clarity, visual appearance, $\mathrm{pH}$ and Drug content}

The prepared formulations were observed for appearance and clarity by visually adjacent to black and white surroundings. The surface $\mathrm{pH}$ of the in situ ocular gels were determined by keeping the digisun digital $\mathrm{pH}$ meter on the formulations and allowed equilibrates for one minutes ${ }^{7}$. For drug content, sample $(1 \mathrm{~mL})$ was allowed to dissolve in $10 \mathrm{mLof}$ STF solution. Aliquot of
$1 \mathrm{~mL}$ was withdrawn and further diluted to $10 \mathrm{~mL}$ with STF. The resultant solution was filtered through filter paper and the amounts of LEV present in the in situ ocular gels are determined using UV Spectrophotometer at $287 \mathrm{~nm}$ (Shimadzu 1800, Japan).

\section{In vitro gelation studies}

The gelling capability of the prepared formulation was determined by introducing a drop of the formulation in a vial containing $2 \mathrm{~mL}$ of freshly prepared STF and 
visually observed. The time taken for its gelling was noted $^{8,9}$.

\section{Rheological studies}

The Brookfield viscometer LVDV-E was used to measure the viscosity of the LEV in situ ocular gels. The in situ gel formulations were placed in the sampler tube. The samples were analyzed by a circulating shower at 37 ${ }^{\circ} \mathrm{C} \pm 0.5{ }^{\circ} \mathrm{C}$ linked to the viscometer adaptor prior to every determinations. The angular velocity of the spindle was increased from 1 to $4 \mathrm{rpm}$ and the viscosity of the formulation was measured ${ }^{10,11}$.

\section{In vitro drug release studies}

Modified diffusion apparatus was used to carry out the in vitro release of LEV from developed in situ ocular gels and STF (pH7.4) was used as a diffusion medium ${ }^{12}$. The soaked cellophane membrane in the diffusion medium for the night, was fixed to one end of a specially designed glass cylinder which was opened at both ends. The LEV ocular gel was accurately placed into the glass cylinder (donor compartment) and this cylinder was immersed in a beaker (receptor compartment) containing $50 \mathrm{~mL}$ of diffusion medium at a $37 \pm 2{ }^{\circ} \mathrm{C}$ with $50 \mathrm{rpm}$, so that the membrane touches the surface of the medium. Sample $(1 \mathrm{~mL})$ was withdrawn at a predetermined time intervals (1, 2, 3, 4, 5, 6, 7 and 8 hours) and replaced with an equal volume of fresh diffusion medium. The aliquots were diluted with the diffusion medium and assayed at $287 \mathrm{~nm}$ using UV Spectrophotometer (Shimadzu 1800, Japan). Parallel $2 \mathrm{~mL}$ of marketed formulation of Levofloxacin $0.5 \%(5 \mathrm{mg} / \mathrm{ml})$ - Quixin were studied in the similar manner. The mechanism of drug release from the ocular gel was determined by finding the best fit of the release data to Higuchi and Korsmeyer-Peppas plots. The release rate constants ' $\mathrm{k}$ ' and ' $n$ ' of each model were calculated by linear regression analysis using Microsoft Excel 2010 software. Coefficients of determination $\left(r^{2}\right)$ were used to evaluate the accuracy of the fit ${ }^{13,14}$.

\section{Sterility}

Direct inoculation method was used to perform the sterility of the prepared formulations. From the test solution $(2 \mathrm{~mL})$ was withdrawn using a sterile pipette and aseptically transferred to fluid thioglycolate medium $(20 \mathrm{~mL})$ and soyabean-casein digest medium $(20 \mathrm{~mL})$ separately. After inoculation the media was incubated for not less than 14 days at $30-35{ }^{\circ} \mathrm{C}$ in the case of fluid thioglycolate medium and $20-25{ }^{\circ} \mathrm{C}$ in the case of soyabean-casein digest medium ${ }^{15}$.

\section{Antimicrobial efficacy studies}

The drug was allowed to diffuse through a solid agar medium. The standard minimum inhibitory concentration (MIC $2 \mu \mathrm{g} / \mathrm{mL}$ ) of LEV and developed formulations containing LEV were prepared. Antimicrobial activity was determined by agar diffusion test employing cup plate technique. Staphylococcus aureus, Pseudomonas aeruginosa were used as the test organisms to study the antimicrobial efficacy (biological activity). The standard minimum inhibitory concentration (MIC $2 \mu \mathrm{g} / \mathrm{mL}$ ) of control and developed formulations $(10 \mu \mathrm{g} / \mathrm{mL})$ containing LEV were prepared. The solutions were poured in to cups bored into sterile nutrient agar previously seeded with test organisms (Pseudomonas aeruginosa, and Staphylococcus aureus). The drug was allowed to diffuse through a solid agar medium. After the diffusion of the solutions for 2 hours, the agar plates were incubated at $37^{\circ} \mathrm{C}$ for 24 hours. The Zone of inhibition (ZOI) was measured around each cup and compared with that of control. The entire operation except the incubation was carried out in a laminar airflow unit. Both positive and negative controls were maintained during the study ${ }^{15}$.

\section{Ocular irritation studies}

Ocular irritation study was performed for selected formulations using male albino rabbits (four), each weighing about 2 to $3 \mathrm{~kg}$. $0.1 \mathrm{~mL}$ of the selected sterile LEV formulation was instilled in to cul-de-sac twice a day for a period of 14 days. The rabbits were monitored periodically for redness, swelling, watering of the eye ${ }^{16}$.

\section{Accelerated stability studies}

Selected sterile LEV formulations were filled in glass vials, closed with gray butyl rubber closures and sealed with an aluminium caps. The vials contain optimized formulation were kept in stability chamber, maintained at $40 \pm 2{ }^{\circ} \mathrm{C}$ and $75 \pm 5 \% \mathrm{RH}$ for one month. Samples were withdrawn weekly and estimated for drug content, $\mathrm{pH}$, visual appearance, gelling capacity and in vitro drug release $^{17}$.

\section{RESULTS AND DISCUSSION}

\section{Evaluation of in situ gels of LEV Hydrochloride}

Total of 8 formulations of in situ gels of Levofloxacin hydrochloride were prepared by $\mathrm{pH}$ triggered in situ gelling system using different polymers like sodium alginate as gelling agent, Noveon AA-1 polycarbophil and HPMC E50LV as viscosity enhancing agent and benzalkonium chloride as preservative.

\section{Appearance, Clarity, Determination of $\mathbf{p H}$ and Drug} content

The visual appearance, clarity and the $\mathrm{pH}$ of the prepared formulations are shown in Table 2. All the prepared formulations were clear and the visual appearance was found to be transparent. Terminal sterilization by autoclaving had no effect on physicochemical properties and the clarity of the formulations. The $\mathrm{pH}$, solubility and stability is one of the most significant factor concerned in the ophthalmic preparation. The two areas of considerable significance are the effect of $\mathrm{pH}$ and stability of the prepared formulation. During the formulation of in situ gels, care has been taken that there should not be any irritation while administration. The pHof the prepared formulations was ranged between 6.50 and 7.00. The drug content varied between $96.84 \pm 0.396 \%$ and $99.65 \pm 0.489 \%$ which indicated that the uniform distribution of drug was found in all the prepared formulations. Among all the formulations, formulation code A4 showed maximum drug content. 
Table 2: Appearance, Clarity, pH and Drug content of in situ ocular gels of LEV

\begin{tabular}{|c|c|c|c|c|}
\hline Formulation Code & Appearance & Clarity & pH $^{*}$ & Drug content $\left.^{(\%)}\right)^{*}$ \\
\hline A1 & Transparent & Clear & 6.70 & $97.57 \pm 0.561$ \\
\hline A2 & Transparent & Clear & 6.62 & $97.96 \pm 0.624$ \\
\hline A3 & Transparent & Clear & 6.93 & $98.64 \pm 0.341$ \\
\hline A4 & Transparent & Clear & 6.98 & $99.65 \pm 0.489$ \\
\hline A5 & Transparent & Clear & 6.50 & $96.84 \pm 0.396$ \\
\hline A6 & Transparent & Clear & 6.82 & $98.12 \pm 0.474$ \\
\hline A7 & Transparent & Clear & 6.78 & $98.61 \pm 0.547$ \\
\hline A8 & Transparent & Clear & 7.00 & $99.09 \pm 0.647$ \\
\hline
\end{tabular}

*Mean \pm S.D, $n=3$

\section{In vitro gelation studies}

Among all the formulations, the formulations A4 and A8 showed better gelling capacity Table 3 . This may be due to the higher uptake capacity of the polymers.

\section{Rheological studies}

The rheological properties (pre gelation and post gelation viscosity studies) of the prepared in situ ocular gels of LEVare shown in Figure 1- 2. The results showed that the viscosity of all the prepared formulations reduced as the shear rate increased, which indicated the character of pseudo plastic fluid. The shear rate on the preparation was large during the blinking stage. If the viscosity is too high, this will result in irritation in the eye and it is too low, it may produce increased drainage. Therefore, the formulation should have optimum viscosity for easy instillation into the eye as liquid, which will go through a rapid sol-to-gel transition, hence the good gelling capacity.

Table 3: Gelling capacity of in situ ocular gels of LEV

\begin{tabular}{|c|c|}
\hline Formulation code & Gelling Capacity \\
\hline A1 & + \\
\hline A2 & + \\
\hline A3 & ++ \\
\hline A4 & +++ \\
\hline A5 & + \\
\hline A6 & + \\
\hline A7 & ++ \\
\hline A8 & +++ \\
\hline +Gels after few minutes and dissolves rapidly
\end{tabular}

++ Gels immediately and remains for few hours

+++ Gels immediately and remains for extended period of time

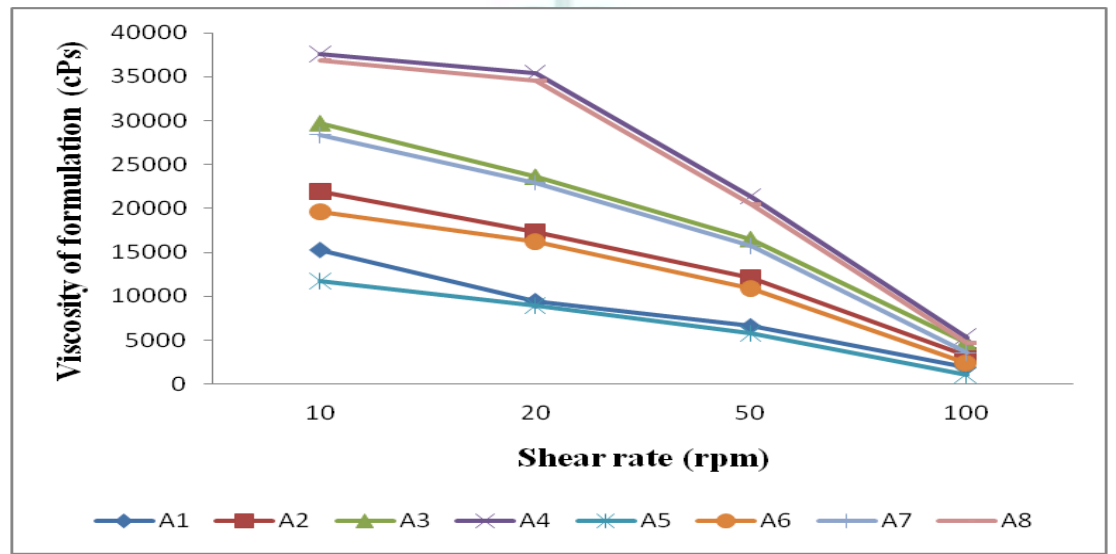

Figure 1: Pre-gelation viscosity studies of in situ ocular gels of LEV

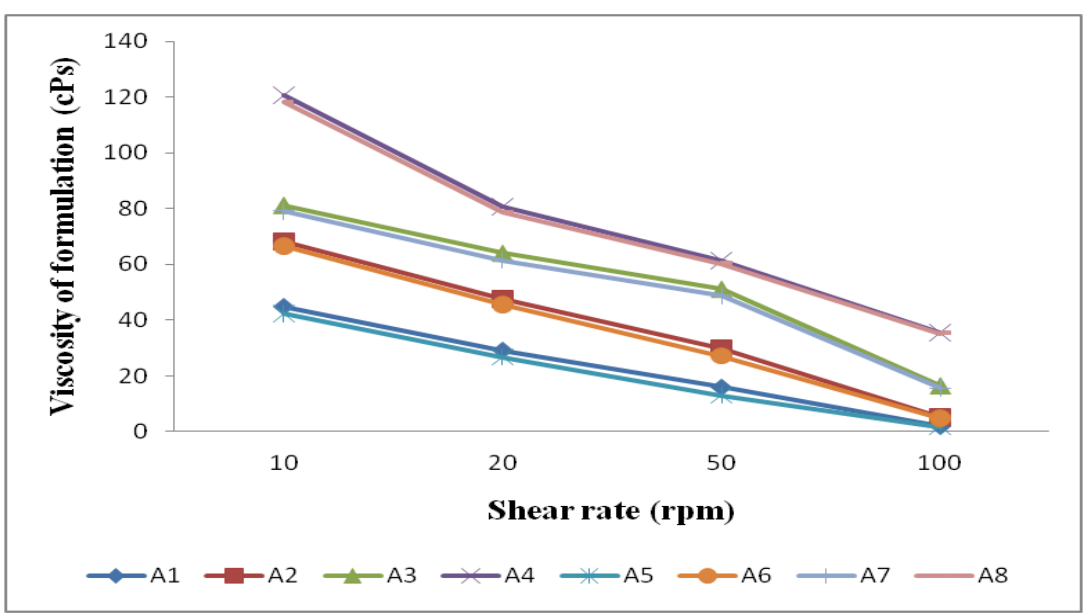

Figure 2: Post-gelation viscosity studies of in situ ocular gels of LEV 


\section{In vitro drug release studies}

The in vitro release of LEV from the in situ ocular gelsis shown in Figure 3. The prolonged drug release was showed by the formulation A8 $98.72 \%$ at a period of $8 \mathrm{~h}$ and followed by the formulation A4 96.19\%. The formulations A3 showed $95.1 \%$ and A7 showed $97.7 \%$ maximum drug release for a period of 7 hours. The formulations A1 showed the maximum drug of release 97.95\%, A2 showed $96.34 \%$, A5 showed $97.13 \%$ and A6 showed $96.54 \%$ after 6 hours. The extended period of drug release may be due to relaxed diffusion of drug from the formulations. The combinations of polymers might have played an important role in diffusion and thereby exhibit controlled drug delivery. Moreover formation of gel matrix between drug and polymers might have helped to attain the rate controlled release. From the in vitro drug release profile the formulations A4 and A8 was selected as the best formulations and these formulations were used for further studies such as mechanism of drug release, sterility, antimicrobial efficacy, ocular irritation and accelerated stability.

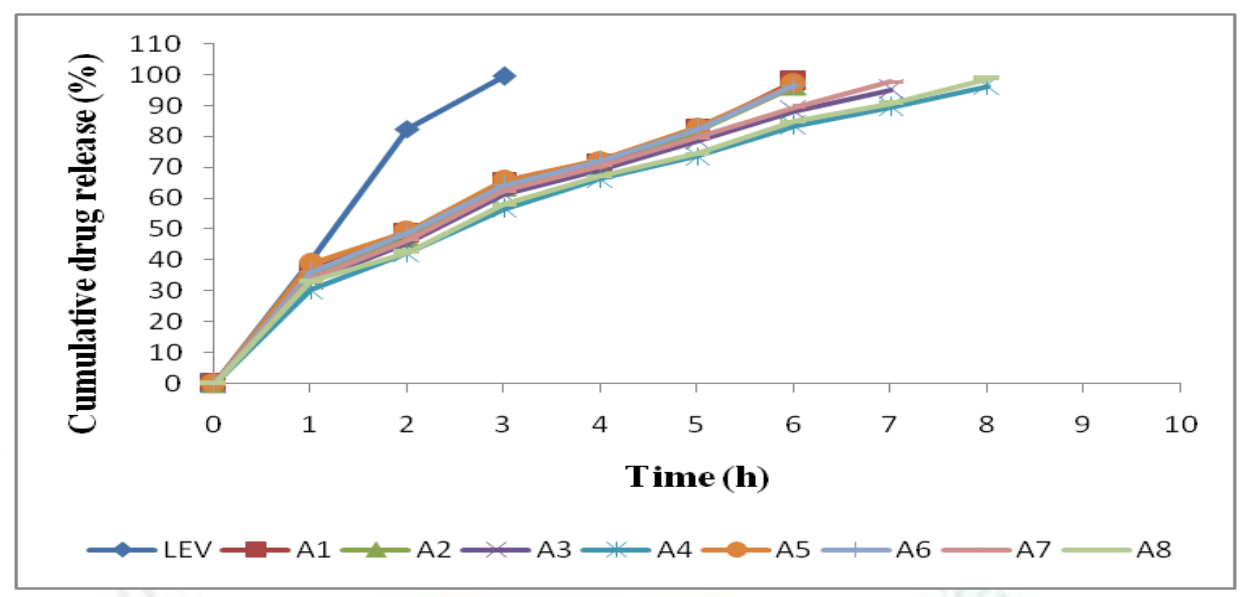

Figure 3: In vitro drug release of the formulations A1 - A8

\section{Mechanism of drug release}

The mechanisms of drug release from the selected in situ ocular gels were determined by finding the best fit of release data to Higuchi and Korsmeyer-Peppas plots. The release rate constant ' $k$ ' and ' $n$ ' of each model were calculated by linear regression analysis. Co-efficient of determination $\left(r^{2}\right)$ was used to evaluate the accuracy of the fit. The $r^{2}, k$ and $n$ values are shown in Table 4.

Table 4: The $\mathbf{r}^{2}, k$ and $n$ values of selected formulations

\begin{tabular}{|c|c|c|c|c|c|}
\hline \multirow{2}{*}{ Formulations } & \multicolumn{2}{|c|}{ Higuchi } & \multicolumn{2}{c|}{ Korsmeyer-Peppas } & \multirow{2}{*}{ Mechanism of drug release } \\
\cline { 2 - 5 } & $\boldsymbol{r}^{\mathbf{2}}$ & $\mathbf{k}\left(\mathbf{h}^{-\mathbf{1}}\right)$ & $\boldsymbol{r}^{\mathbf{2}}$ & $\mathbf{n}$ & \\
\hline $\mathbf{A 4}$ & 0.9984 & 0.2552 & 0.9976 & 0.4788 & Diffusion \\
\hline $\mathbf{A 8}$ & 0.9982 & 0.2883 & 0.9971 & 0.4979 & Diffusion \\
\hline
\end{tabular}

Both formulations provided good fit to the Higuchi model. According to this model, the drug release from these gels may be controlled by diffusion through the micropores.

\section{Sterility and Antimicrobial efficacy and Ocular irritation studies}

The sterility studies indicated that the selected formulations were sterile when incubated for a minimum of 14 days at $30-35{ }^{\circ} \mathrm{C}$ in case of fluid thioglycolate medium and at $20-25^{\circ} \mathrm{C}$ in the case of soya bean-casein digest medium. The antimicrobial efficiency of the selected formulation is shown in Table 5. After incubation, the result indicated that the selected formulations showed good anti-microbial action against the organisms. The ocular irritation studies revealed that the selected formulations were good with non-irritation and there were no ocular damage or abnormal clinical signs.

Table 5: Antimicrobial studies of the selected formulations

\begin{tabular}{|c|c|c|c|}
\hline \multirow{2}{*}{ Test micro organisms } & \multicolumn{3}{|c|}{ Diameter of the zone of inhibition produced by in situ gels (mm) } \\
\cline { 2 - 4 } & LEV & A4 & A8 \\
\hline Staphylococcus Aureus & 26 & 26 & 25 \\
\hline Pseudomonas Aeruginosa & 33 & 33 & 33 \\
\hline
\end{tabular}




\section{Accelerated stability studies}

During and at the end of the accelerated stability study, the selected formulations did not undergo any chemical changes/interaction and remained stable during the study period and showed almost similar physical stability and drug content.

\section{CONCLUSION}

LEV is a broad spectrum anti bacterial agent used in the treatment of various ocular infections. In situ ocular gels of LEV were prepared and in vitro drug release indicated that it is potential drug delivery of LEV. The optimized formulations (A4 and A8) showed good antibacterial efficacy with non irritant character. In vivo studies are warranted to confirm these results in future.

\section{REFERENCES}

1. Gupta S, Suresh PV ,Carbopol/Chitosan based $\mathrm{pH}$ triggered in situ gelling system for ocular delivery of Timolol Maleate,Sci Pharm, 2010; 78:959-976.

2. Rajas NJ, Kavitha K, Gounder T, Mani T,In situopthalmic gels: a developing trend,Int J Pharma Sci Rev Res, 2011; 7(1):8-14.

3. Rathore KS, In situ gelling ophthalmic drug delivery system: an overview, Int J Ph Pharma Sci, 2010, 2(4): 30-34.

4. Mohanambal E, Arun K,SathaliHA, Formulation and Evaluation of pH-triggered in situ Gelling System of Levofloxacin, Ind J Pharm Edu Res, 2011; 45(1):58-64.

5. Diren S, Zeynep FK,Bioavailability File: Levofloxacin, $J$ PharmSci, 2007; 32:197-208.

6. Nayak NS, Bharani SS, Thakur RS, Formulation and evaluation of $\mathrm{pH}$ triggered in situ ophthalmic gel of Moxifloxacin hydrochloride,Int J Pharm PharmSci, 2012; 4(2):452-459.
7. Srividya, Rita MC, Amin PD, Sustained ocular delivery of Ofloxacin from a $\mathrm{pH}$ activated in situ gelling system, J Control Rel, 2001; 73:205-211.

8. Gokulgandhi MR, Parikh JR, Barot MM, Modi DM. A pH activated in situ gel forming ocular drug delivery system used for tropicamide, Drug Delivery Technology, 2007; 5:44-49.

9. Zhidong L, Jiawei L, Shufang N, Hui L, Pingtian D, Weisan P, Study of HPMC/alginate based in situ gelling ocular delivery system for gatifloxacin. Int J Pharm, 2006; 315:1217.

10. Indu PK, Manjit S, Meenakshi K, Preparation and evaluation of ocular formulations of acetazolamide, Int J Pharm, 2000; 199:119-127.

11. Pandit D, Bharathi A, Srinatha R, Singh S, Long acting ophthalmic preparations of indomethacin: Assessment of gel systems of alginate, Indian J Pharm Sci, 2007; 69:37-40.

12. Mandal S, Manjunath KMJ, Thimmasetty M, GL Prabhushankar, Geetha MS, Preparation and evaluation of an in situ gel forming ophthalmic preparations of moxifloxacin hydrochloride, International Journal of Pharmaceutical Investigation, 2012; 2 (2):78-82.

13. Higuchi $T$, Rate of release of medicaments from ointment bases containing drugs in suspension, J PharmSci, 1961; 50:874-875.

14. Korsmeyer RW, Gurny R, Doelker E, Buri P and Peppas NA, Mechanism of potassium chloride release from compressed hydrophilic polymeric matrices: effect of entrapped air, JPharmSci, 1983; 72(10):1189-1191.

15. Controller of Publication, Indian Pharmacopoeia, Ministry of Health and Family Welfare, Government of India, New Delhi, 2007.

16. Draize J, Woodward G, Calvery O, Methods for the study of toxicity and irritation of substance applied locally to the skin surface and mucous membrane, J Pharm Col ExpTher, 1994; 82:377-390.

17. Mathews BR, Regulatory features of stability testing in Europe, Drug Dev Ind Pharm, 1999; 25:831-856. 